\title{
Forschendes Lernen im Bereich der rechtlichen Fragen der Erneuerbaren Energien - ein Werkstattbericht am Beispiel der Hochschule für öffentliche Verwaltung Kehl
}

\author{
Michael Frey"
}

\section{A. Einleitung: Forschendes Lernen - Begriff und Eignung für die Nutzung an Hochschulen für angewandte Wissenschaften (HAW)}

\section{Der Begriff des Forschenden Lernens}

Der Begriff des Forschenden Lernens taucht heute aufgrund seiner Eingängigkeit vielfach in der Beschreibung von Projekten und Curricula von Hochschulen auf. ${ }^{1}$ Zweck des Forschenden Lernens ist eine aktive und selbstständige Beteiligung von Studierenden an der Forschung selbst.

Der Begriff wurde maßgeblich von der Bundesassistentenkonferenz 1970 geprägt $^{2}$ und kann mit folgenden Merkmalen umrissen werden: Die Studierenden sollen

- anhand eines (möglichst) selbst gewählten Themas

- auf der Grundlage einer eigenständig entwickelten Strategie in Bezug auf Methodenwahl, Versuchsanordnungen oder Recherche mit dem entsprechenden Risiko des Scheiterns aber auch den Chancen für Zufallsentdeckungen

- dem Anspruch der Wissenschaft genügende Forschungsergebnisse, die neu sind oder zumindest für Dritte von Interesse sind erarbeiten und

- diese so darstellen, dass ihre Bedeutung erkennbar und ihre Herleitung nachvollziehbar wird. ${ }^{3}$

Hieraus entwickelt Huber den Versuch einer eingängigen Definition, welche für den weiteren Text zugrunde gelegt werden soll:

„Forschendes Lernen zeichnet sich vor anderen Lehrformen dadurch aus, dass die Lernenden den Prozess eines Forschungsvorhabens, das auf die Gewinnung von auch für Dritte interessanten Erkenntnissen gerichtet ist, in seinen wesentlichen Phasen - von der Entwicklung der Fragen und Hypothesen über die Wahl und Ausführung der Methoden bis zur Prüfung und Darstellung der Ergebnisse in selbständiger Arbeit oder in aktiver Mitarbeit in einem übergreifenden Projekt (mit)gestalten, erfahren und reflektieren."

* Der Autor ist seit 2013 Professor der Hochschule Kehl an der Fakultät I Rechts- und Kommunalwissenschaften.

1 Huber, in: ders./Hellmer et al. (Hrsg.), S. 9 ff.

2 Denkschrift der Bundesassistentenkonferenz, zitiert nach Huber, in: ders./Hellmer et al. (Hrsg.), S. 9 ff.

3 Denkschrift der BAK, ebd.

4 Huber, in: ders./Hellmer et al. (Hrsg.), S. 9 (11). 


\section{Forschendes Lernen an HAW - Widerspruch oder Chance?}

Aus universitärer Sicht mag auf den ersten Blick die anwendungsbezogene Grundausrichtung der Hochschulen für angewandte Wissenschaften einer umfassenden Anwendung des Forschenden Lernens entgegenstehen.

Allerdings werden die HAW bereits heute in den Hochschulgesetzen der Länder dazu ermächtigt und verpflichtet, anwendungsbezogen zu forschen. ${ }^{5}$ Die vielfach noch anzutreffende Vorstellung einer (reinen) Lehrhochschule in Abgrenzung zur forschenden Universität ist insoweit nicht nur de lege lata sondern auch in praxi überholt. ${ }^{6}$

Vielmehr bieten gerade auch die Rahmenbedingungen an HAW zumindest teilweise günstige Voraussetzungen für eine Anwendung Forschenden Lernens. Anzuführen ist hier insbesondere die in der Regel gute Laborausstattung in den technischen Studiengängen, generell aber auch die noch immer geringere Gruppengröße und damit ein besseres und unmittelbareres Verhältnis zwischen Professoren und Praktikern einerseits und Studierenden andererseits.

Freilich lassen sich auch hemmende Rahmenbedingungen feststellen, wie höhere Lehrverpflichtungen der Professoren an HAW, ein oft nur rudimentär ausgeprägter wissenschaftlicher Mittelbau und häufig noch starr auf Kompetenzvermittlung ausgerichtete Curricula mit meist einsemestrigen Modulen, die bisweilen nur wenig zeitlichen und inhaltlichen Freiraum für eine Umsetzung Forschenden Lernens lassen. ${ }^{7}$ Aus der Sicht der HAW betrachtet, sind damit jedoch zugleich die wesentlichen Stellschrauben genannt, an denen gedreht werden muss, will man die Anteile Forschenden Lernens an HAW erhöhen.

Der vorliegende Text stellt an einem Beispiel aus dem Studiengang „Gehobener Verwaltungsdienst - Public Management" der Hochschule Kehl vor, wie Forschendes Lernen bereits in bestehenden Lehrveranstaltungen, hier sog. Proseminaren und Fachprojekten, integriert werden kann.

\section{B. Das Beispiel der Hochschule Kehl - Studienablauf, Möglichkeiten für die Integration des Forschenden Lernens in den Studienablauf}

\section{Die Hochschule Kehl und der Studienablauf, geeignete Module für die Integration Forschenden Lernens}

Die Hochschule für öffentliche Verwaltung Kehl bildet in erster Linie Beamte und Beschäftigte für den gehobenen Verwaltungsdienst des Landes und für Schlüsselpositionen in Kommunen aus. Das insgesamt sechs Semester dauernde Bachelor-Studium „Gehobener Verwaltungsdienst - Public Management“ gliedert sich in ein drei Semester dauernden Grundstudium, eine einjährige Praxisphase und ein abschlie-

5 Z.B. $\$ 2$ Abs. 1 Nr. 4 LHG BW.

6 Vgl. hierzu: http://www.che.de/downloads/CHE_AP_171_FH_Forschung.pdf (29.5.2014).

7 Insoweit auch generell: Huber, in: ders./Hellmer et al. (Hrsg.), S. 9 (22). 
ßendes Vertiefungssemester an der Hochschule. ${ }^{8}$ Dem Studium vorgeschaltet ist ein verpflichtendes einsemestriges Einführungspraktikum, das in der Regel bei einer Kommune absolviert wird.

In der Studien- und Prüfungsordnung 9 und dem diese konkretisierenden Modulhandbuch der Hochschule für diesen Studiengang werden für den Bachelor-Studiengang „Gehobener Verwaltungsdienst - Public Management“ Fachprojekte und Proseminare für das zweite und dritte Fachsemester mit einer Laufzeit von zwei Semestern und einem Stundenansatz von drei Semesterwochenstunden angesetzt. ${ }^{10}$

Bereits heute werden an der Hochschule im Rahmen des Grundstudiums Fachprojekte und Proseminare aus dem Bereich der Erneuerbaren Energien mit großem Erfolg und hoher Nachfrage von studentischer Seite angeboten. Beide Lehrformen erscheinen zwar grundsätzlich als Ausgangsbasis für Forschendes Lernen gleichermaßen geeignet, während das Proseminar in erster Linie das Erlernen des wissenschaftlichen Arbeitens bezweckt, steht bei einem Fachprojekt die Bearbeiten einer konkreten Fragestellung in einem Team im Vordergrund. Insofern erscheint das Proseminar prädestiniert für die Integration des Forschenden Lernens.

\section{Die praktische Umsetzung an der Hochschule Kehl am Beispiel des Themas „Ausbau der Erneuerbaren Energien“}

Um den Studierenden die maximal mögliche zeitliche Flexibilität und damit auch die Möglichkeit einer von den Prüfungsphasen entkoppelten Forschungstätigkeit zu geben, beginnt die Arbeit für die Studierenden an der Hochschule Kehl in diesem Proseminar bereits unmittelbar nach der Wahl der Proseminare/Fachprojekte, auf jeden Fall noch im ersten Semester des Grundstudiums (Sommersemester). Dadurch gewinnen die Studierenden zumindest die anschließenden Semesterferien zum Einlesen und für Recherchearbeiten.

Ein Beginn des Forschenden Lernens im ersten Fachsemester erfordert von den Lehrenden ein erhöhtes Maß an Grundlagenarbeit. Im Rahmen eines Startworkshops, des sogenannten „Milestone 0-Treffens“ müssen, bevor die Forschungsthemen ausgewählt werden können, die großen Strukturen des Themenbereichs, im vorliegenden Fall der Zusammenhänge der Energiewende einschließlich der Planungs- und Zulassungsverfahren sowie die wesentlichen Grundlagen und Methoden des wissenschaftlichen Arbeitens erläutert werden.

Ebenfalls noch in diesem Semester finden erste gemeinsame Praxisbesuche vor Ort in Energievorreitergemeinden des Landes mit einer Einführung/Problemdarstellung aus der Sicht der handelnden Gemeinde (und damit des zukünftigen potentiellen Ar-

8 Details vgl. http://www.hs-kehl.de/studierende/bachelor/studienablauf/ (29.5.2014).

9 \7 SPO, http://www.hs-kehl.de/fileadmin/hsk/Hochschule/Dokumente/PDF/Studien-_und_Pruefungs ordnung_SPO_fuer_den_BA_i.d._F._der_5._AEnderungssatzung_v._20.2.2012.pdf (29.5.2014).

10 http://www.hs-kehl.de/fileadmin/hsk/Hochschule/Dokumente/PDF/Modulhandbuch_Grundlagenstu dium_Stand_14.6.2012.pdf (29.5.2014).; http://de.wikipedia.org/wiki/Hochschule_Kehl (29.5.2014). 
beitgebers) statt. Die Studierenden werden darüber hinaus ab diesem Zeitpunkt in die regelmäßig an der Hochschule stattfindenden „Praktikerstammtische“ eingebunden. Ziel dieser Maßnahmen ist einerseits ein frühzeitiges Heranführen der Studierenden an die Erwartungen der Praxis, andererseits den Studierenden Forschungsthemen mit Praxisrelevanz zu vermitteln. ${ }^{11}$

Die Wahl der Themen durch die Studierenden erfolgt in einem zweistufigen Verfahren: In der ersten Stufe werden die jeweiligen inhaltlichen Prioritäten der Studierenden anhand von inhaltlichen Kategorien (wie z.B. Windenergie, Wasserkraft, Geothermie, Netze, Fördermittel und Kampagnen) festgestellt. In einem zweiten Schritt werden dann die von den Lehrenden als geeignet erachteten oder von Praktikern vorgeschlagenen Themen der jeweiligen inhaltlichen Kategorien präsentiert, aus denen die Studierenden auswählen können. Es hat sich in der Praxis empfohlen, die Themen von Studierenden in Zweierforschungsteams bearbeiten zu lassen. Dafür stehen nun noch zwei volle Semester einschließlich der Semesterferien zur Verfügung.

In regelmäßigen, meist im 14-tages-Rhythmus stattfindenden „Milestone-Treffen“ innerhalb der gesamten Forschergruppe, aber auch gegenüber der Praxis bei den „Praktikerstammtischen“ berichten die Studierenden über den Stand und den Fortschritt der Arbeiten, aber auch über Schwierigkeiten. Ziel der Milestone-Treffen ist ein regelmäßiges Peer-Feedback einerseits sowie eine möglichst frühzeitige, bereits forschungsbegleitende Nutzbarkeit der entstehenden Erkenntnisse durch die Praxis. Gleichzeitig können durch die Milestone-Treffen die Bedeutung wissenschaftlicher Standards und deren Einhaltung und Umsetzung im eigenen Text vermittelt werden.

$\mathrm{Zu}$ Beginn des Proseminars haben die Studierenden erst ein Semester hinter sich, so dass viele von den Forschungsbereichen für die Studierenden größtenteils unbekannt sind. ${ }^{12}$

Bewährt hat sich daher eine Einlesephase während der Semesterferien mit der Verpflichtung zur Abgabe einer aussagekräftigen vorläufigen Gliederung zu Beginn des zweiten Semesters. Diese Gliederung wird in Individualtreffen mit den Lehrenden besprochen und anschließend kurz nach Semesterbeginn vor der Gruppe präsentiert. Für den Beginn der darauffolgenden Umsetzungsphase hat sich weiterhin eine anwendungsbezogene Auffrischung der wissenschaftlichen Standards mit Praxistipps zu Formulierungen und Formalia im Rahmen eines „Formalia-Workshops“ als nützlich herausgestellt.

11 Die Bezeichnung „Praktikerstammtische“ wurde gewählt, um zum einen die Freiwilligkeit der Teilnahme, zum anderen auch die Möglichkeit zur Diskussion auf Augenhöhe und „ins Unreine“ zu verbalisieren. Ziel ist es, eine Veranstaltung zum Austausch zwischen Studierenden, Wissenschaft und Praxis unter Einbeziehung der Studierenden mit möglichst niedriger Hemmschwelle zu schaffen. Diese Veranstaltungsform dient zur Steuerung des Projekts und zur Zwischenpräsentation der Forschungsfortschritte.

12 An der Hochschule Kehl werden hier die Grundlagen der Rechts- und Verwaltungswissenschaften gelehrt. Dies hat zur Folge, dass wesentliche Bereiche erst zu einem späteren Zeitpunkt behandelt werden. Dies betrifft im vorgestellten Projekt insbesondere die Felder des Besonderen Verwaltungsrechts (Bau- und Immissionsschutzrecht), die erst im dritten Semester durchlaufen werden. Folglich stehen die Studierenden vor einem ihnen größtenteils unbekannten Rechtsgebiet. 
Während der Folgezeit erfolgt die wissenschaftliche Begleitung neben den MilestoneTreffen, die während der klausurintensiven Perioden reduziert werden können, ergänzend mit individuellen Arbeitstreffen zwischen Forschergruppen und Lehrenden sowie im Rahmen der Praktikerstammtische.

Im Semesterverlauf soll dann ein Rohtext bis zum Ende des Wintersemesters erstellt werden. Dieser wird anschließend von den Lehrenden durchgesehen und in den Semesterferien zwischen Winter- und Sommersemester überarbeitet. Dabei soll der fertige Text zunächst in der für die Bachelorarbeit vorgegebenen Form erstellt und abgegeben werden, um den Studierenden die hierfür erforderlichen Kenntnisse zu vermitteln und die Weiternutzung der Formatvorlage für die spätere Bachelorarbeit zu ermöglichen. Diese Arbeit bildet den wesentlichen Teil der Bewertung des Proseminars. Da die Arbeit in Zweierteams erstellt wurde, muss der Text eine Angabe enthalten, welcher Textteil welchem Autor zugeordnet werden soll. Je nach Bedarf steht auch noch ein Puffer innerhalb der ersten vier Semesterwochen des Sommersemesters zur Verfügung.

In Rahmen eines gemeinsamen Klausurwochenendes im Sommersemester sollen die Texte anschließend für die wissenschaftliche Veröffentlichung vorbereitet werden. Hierbei sollen die Studierenden die Schritte zu einer erfolgreichen Publikation durchlaufen und die Anforderungen an einen interessanten wissenschaftlichen Text erkennen. Diese sodann nochmals durchgesehenen Texte werden anschließend den Schriftleitungen entsprechend passenden wissenschaftlichen Fachzeitschriften unterbreitet. Als ergänzende Betreuungsform kann auch ein studentisches Peer-ReviewVerfahren zum Einsatz kommen, bei dem die Studierenden ihren Text einer anderen Forschergruppe aus dem Proseminar zum „Gegenlesen“ geben.

Außerdem soll in diesem Semester der jahrgangsübergreifende Transfer mit dem nachfolgenden Proseminar mittels einer Abschlussveranstaltung sichergestellt werden, bei der die Forschungsergebnisse der Hochschulöffentlichkeit präsentiert werden, denn nach Ablauf dieses Semesters gehen die Studierenden in die einjährige Praxisphase. Zu diesem Zweck nutzen die Studierenden Facebook und die Praktikerstammtische, die auch von ehemaligen Proseminarteilnehmern besucht werden.

Erfreulich an diesem nunmehr drei Jahre laufenden Verfahren ist zum einen, dass praktisch alle Texte auch zur Veröffentlichung angenommen wurden, ${ }^{13}$ außerdem haben viele der Studierenden anschließend Teile ihrer Praxisphase in themennahen Teilen der Verwaltung absolviert, etwa in Gemeinden oder beispielsweise beim Kompetenzzentrum Energie des Regierungspräsidiums Freiburg und konnten dort von

13 Studienjahr 2012/13: Kaufmann/Knobelspies, in: ZNER 2013, S. 250 ff.; Bruckert/Lechleiter, in: VR 2013, S. 374 ff.; Pusch/Witzig, in: innovative VERWALTUNG 2013, 9/2013, S. $41 \mathrm{ff}$.; Herbst, in: VR 2013, S. 336 ff.; Franco/Damm, in: 2013, S. 327 ff.; Holzapfel/Müller, in: VR 2013, S. 233 ff.; aus Studienjahr 2013/14: Zachmann/Späth, in: VR 2014, i.E.; Rothardt/Burkhardt, in: ZfBR 2014, i.E.; Schifferdecker, in: NuR 2014, i.E.; Schmidt/Roth, in: apf 2014, 295; Steible/Grothe, in: VBlBW 2014, i.E.; Fritz/Moll, in: VR 2014, 338; Frey/Staiger, in: apf 2014, 299; Miller/Reichert, in: Wassertriebwerk 2014, 110. 
ihrer Erfahrung profitieren und diese einbringen. ${ }^{14}$ Mehrere Studierende beschäftigten sich weiter in der Forschung und haben in der Folge noch während der Praxisphase weitere wissenschaftliche Texte verfasst und veröffentlicht. ${ }^{15}$

Insofern sind die grundsätzliche Durchführbarkeit des Ansatzes, das Interesse der Studierenden daran und die Praxisrelevanz bereits erwiesen.

\section{Fachliche Ziele des verfolgten Konzepts}

Mit der Umsetzung des Konzepts des Forschenden Lernens zu aktuellen Fragen des Ausbaus der Erneuerbaren Energien im Rahmen von Fachprojekten und Proseminaren an der Hochschule für öffentliche Verwaltung in Kehl sollen mehrere fachliche Ziele verfolgt werden:

Eine prozessbegleitende Anwendungsforschung während laufender Planungs- und Genehmigungsprozesse und die enge Verzahnung mit den Praktiker-Stammtischen sollen dazu beitragen, dass ungeklärte Anwendungsfragen schneller und vor allem praxisgerecht gelöst werden können. Dies kommt insbesondere den Praktikern, im vorliegenden Fall den Planungs- und Genehmigungsbehörden, zugute.

Durch die Einbindung von Studierenden in den eng mit der Praxis vernetzten Forschungsprozess sollen Absolventen hervorgebracht werden, die praxisnah und auf dem aktuellen Stand der Anwendungsforschung sind und die das insbesondere in diesem dynamischen Themenbereich häufig bestehende Know-how-Defizit ausgleichen können. Durch die Organisation als semesterübergreifendes lernendes Forschungsteam mit Chance auf wissenschaftliche Publikationen arbeiten die Studierenden auf der Grundlage einer hohen intrinsischen Motivation. Gleichzeitig wird auch die Fähigkeit zur Zusammenarbeit in Projektteams gefördert.

Innovativ an diesem Modell ist

- die Umsetzung von forschendem Lernen (und lernendem Forschen) an HAW bereits im Rahmen des Grundstudiums unter breiter Einbeziehung von Forschung, Ausbildung und Praxis;

- die jahrgangsübergreifende Verknüpfung der jeweiligen Fachprojekte und Proseminare, durch die auch der fachbezogene Wissenstransfer unter den Studierenden gestärkt wird. Hierdurch entsteht an der Hochschule mittelfristig ein ständige jahrgangsübergreifender Know-How-Cluster zum Ausbau Erneuerbarer Energien, bestehend aus interessierten Studierenden mehrerer Studienjahre einschließlich interessierter Lehrender und Praktiker. Dadurch kann die begrenzte Ausstattung der HAW zumindest teilweise kompensiert werden;

- die Möglichkeit zur Erweiterung dieses durch den verwaltungswissenschaftlichen Zuschnitt der Hochschule Kehl in erster Linie so ausgerichteten Clusters mit technisch orientierten HAW, etwa der Hochschule Offenburg, um die anwendungs-

14 Bäuerlein, Es profitiert nicht allein der Verwaltungsnachwuchs, in: Staatsanzeiger für Baden-Württemberg vom 25.4.2014, S. 6.

15 Herbst, in: VR 2014, 192; Franco/Frey, in: BauR 2014, 1088. 
begleitende Forschung auf technische Aspekte (insbesondere im Rahmen von Anlagenzulassungsverfahren) auszudehnen; hier kommen insbesondere auch interdisziplinäre Forschungsteams in Frage;

- die unmittelbare Nutzbarkeit der gewonnenen Erkenntnisse durch die Praxis noch vor letztlichen Veröffentlichung der wissenschaftlichen Erkenntnisse. ${ }^{16}$ Dies ist vor allem in dynamischen Entwicklungsfeldern mit Bedarf an begleitender Anwendungsforschung von entscheidender Bedeutung.

- Die infolge der frühzeitigen Forschungsmöglichkeit gegebene Chance für die Studierenden, die gewonnenen Erkenntnisse im Rahmen des weiterführenden Studiums (Praktikumsphase, Wahl der Bachelorarbeitsthemen) vertiefen zu können.

- Die Maßnahme führt zu einer Verbreiterung und Verbesserung des Wissenstransfers zwischen den beteiligten Akteuren, was zukünftig noch besser aufeinander abgestimmte Forschungsreihen zu bestimmten Themenbereichen, etwa im Rahmen von in späteren Studienabschnitten folgenden Bachelorarbeiten, ermöglichen soll.

- Unabhängig davon erleichtert das Vorgehen die in den Rechts- und Verwaltungswissenschaften für Studierende und Praktiker bedeutsame Kompetenz zur Herangehensweise an völlig fremde Sach- und Rechtsgebiete.

\section{Didaktisches Ziel und eingesetzte (fachdidaktische) Methoden}

Didaktisches Ziel des Konzepts ist die Vermittlung vertiefter fachlicher Kenntnisse im interdisziplinären Feld des Ausbaus der Erneuerbaren Energien unter möglichst frühzeitiger Anwendung der Grundsätze des Forschenden Lernens anhand von praxisrelevanten Fragen und unter intensiver Einbeziehung der Praxis. Dies soll - unterstützt durch Praktiker und Lehrende - weitestgehend eigenständig bzw. in Teamarbeit zum selbständigen Erarbeiten von Kenntnissen in bislang unbekannten Gebieten führen.

Hierzu wurden folgende Methoden eingesetzt: ${ }^{17}$

Im Rahmen des Startworkshops (Grundstruktur des Themenfeldes, wissenschaftliche Standards und Ermittlung der Forschungsinteressen der Studierenden) kommen sowohl der klassische Vortrag, aber auch das Lernen am Fall, Mindmaps und die Netzplantechnik zum Einsatz.

Seitens der Studierenden sind eigenständige Gruppeneinteilung und -arbeit sowie Entwicklung der Veranstaltung in der Folgezeit gefordert. Hierfür teilen sich die Studierenden Aufgaben zu, die sie eigenverantwortlich während des Proseminars erledigen (wie z.B. die Organisation der Klausurtagung oder der Milestone-Treffen). Denkbar wäre in diesem Zusammenhang auch der Einsatz von Zielvereinbarungen zwischen Lehrenden und Lernenden.

16 Siehe Bäuerlein, Es profitiert nicht allein der Verwaltungsnachwuchs, in: Staatsanzeiger für BadenWürttemberg vom 25.4.2014, S. 6.

17 Vgl. zu Methoden beim Forschenden Lernen: Huber, in: ders./Kröger (Hrsg.), S. 247 ff.; sowie allgemein zu den Methoden: Behrend/Voss et al. (Hrsg.), Neues Handbuch der Hochschullehre, Kapitel C. 
Die Praktiker-Stammtische haben den Zweck der Schaffung einer für die Praxis möglichst niederschwelligen Veranstaltungsform. Hier werden Praxisprobleme auf Augenhöhe mit den Studierenden und interessierten Kollegen aus der Hochschule diskutiert und die Forschungsfortschritte und -ergebnisse der Studierenden gegenüber der Praxis dargestellt. Hierbei kommt als Mikromethode neben dem Bericht auch die Peer-Feedbackmethode zum Einsatz. Im Rahmen der Forschungsbegleitung durch regelmäßige Milestone-Treffen mit Berichten über Erkenntnisse und Probleme innerhalb der Forschungsgruppe und zur Vermittlung von wissenschaftlichen Standards am eigenen Text können auf Mikromethodenebene eingesetzt werden: Blitzlicht oder Mindmapmethode sowie die Leittext-Methode. Beim Klausur-Workshop mit individueller Betreuung zur Textfinalisierung und Herstellung des jahrgangsübergreifenden Bezugs kann darüber hinaus auch noch die World-Café-Methode ${ }^{18}$ eingesetzt werden. Peer-Review-Verfahren, wie sie bei namhaften Zeitschriften zum Einsatz kommen, können durchgespielt werden. Durch die weitergehende Integration von Studierenden der Vorgängerjahrgänge, die den Studierenden für Fragen aller Art zur Seite stehen, etwa bei den Praktikerstammtischen und dem Klausurwochenende findet gleichzeitig auch eine tutorielle Betreuung statt.

Letztlich handelt es sich um eine besondere Form des Team-Teachings, bestehend aus einem Professor der HAW, einem Praktiker und studentischen Hilfskräften der Vorjahre. Aus dieser Kombination resultiert eine effektive Berücksichtigung verschiedenster Interessen um Methodik und Inhalte kritisch und bestmöglich für die Studierenden anzupassen. Eine besondere Symbiose ergibt sich aus der Teilnahme der studentischen Hilfskräfte.

Die „klassische Vorlesung“ wird hingegen vermieden. Stattdessen erfolgt eine Förderung auf der Grundlage einer handlungsorientierten Lehre. Rechtsmethodische Grundlage für die Forschung ist jeweils die juristische Fallmethode.

\section{Einige Anmerkungen zum Transferpotential des vorgestellten Ansatzes}

Hochschulen für öffentliche Verwaltung sind im Rahmen der HAW-Landschaft Sonderfälle, sowohl was ihre fachliche Ausrichtung anbelangt (Lehre schwerpunktmäßig in rechts-, wirtschafts-, finanz- und sozialwissenschaftlichen Fächern), als auch was ihre Größe und den Status ihrer Studierenden anbelangt.

Gleichwohl bietet der gewählte Ansatz ein hohes Transferpotential.

Zunächst kann dieser Ansatz nahezu unverändert auf diesen oder auf andere Themenbereiche mit hoher Aktualität an Hochschulen für öffentliche Verwaltung oder vergleichbaren Lehrschwerpunkten übernommen werden.

Darüber hinaus bietet die Sonderrolle der Hochschulen für öffentliche Verwaltung einen weiteren Transferansatz. Durch ihre verwaltungswissenschaftliche Grundaus-

18 Eine von Juanita Brown und David Isaacs entwickelte Workshop-Methode, siehe dazu etwa www.theworldcafe.com (24.7.2014). 
richtung sind sie zu nahezu allen anderen HAW in diesen Themenbereichen komplementär und kommen damit als ideale Kooperationspartner, insbesondere für naturwissenschaftliche oder ingenieurwissenschaftlich geprägte Hochschulen in Betracht. In der interdisziplinären Vernetzung zwischen diesen HAW liegt damit ein enormes Potential um technische Fragestellungen frühzeitig bereits auch planungsund genehmigungsrechtlich zu erforschen, was in zahlreichen Schlüsselbereichen der Gesellschaft (wie Demografie und Nachhaltigkeit, Energiewende) ein entscheidender Erfolgsfaktor ist. Eine entsprechende Vernetzung könnte mittelfristig zur Bildung eines Recherche- und Forschungsclusters mit einer mehrdimensionalen Vernetzung im Bereich der Studierenden, der Lehrenden, aber auch der Praktiker auf rechtlichadministrativer Seite und Unternehmensseite führen.

\section{Messbarkeit des Erfolgs des didaktischen Konzepts}

Der Erfolg des didaktischen Konzepts kann sich zunächst unmittelbar anhand von Output-Indikatoren, etwa der Anzahl der wissenschaftlichen Veröffentlichungen aus den entsprechenden Modulen von oder mit den Studierenden, aber auch in der Anzahl der ausgebildeten Studenten im entsprechenden Modul messen lassen, zusätzlich könnten auch die in diesem Bereich geschriebenen Bachelorarbeiten als Indikator dienen. Mittelfristig könnten als Outcome-Indikatoren über ein Alumni-Netzwerk die Anzahl der beruflich mit diesen Fragen befassten und in entsprechenden Fachprojekten und Proseminaren ausgebildeten Studierenden erhoben werden.

Denkbar wäre auch, die Anzahl der Folgeveröffentlichungen dieser Studierenden ins Verhältnis zu der Anzahl der Veröffentlichungen der anderen Studierenden zu setzen, um die langfristige Forschungsmotivation der Studierenden zu messen.

\section{E. Fazit}

Mit dem dargestellten Ansatz kann auf der Grundlage bereits existierender curricularer Voraussetzungen ein Modell für Forschendes Lehren an HAW entwickelt werden, bei dem die Forschung, insbesondere aber Praktiker und Studierende einen unmittelbaren Nutzen erzielen können. Durch diesen Ansatz entsteht eine Win-winwin-Situation: Die Praxis gewinnt durch eine schnellere Lösung von Anwendungsproblemen durch die um die Studierenden erhöhte Forschungs- und Problemlösungskapazität, die Studierenden gewinnen Forschungs-Know-How und praxisrelevantes Wissen unter hoher Eigenmotivation sowie Kontakte zu potentiellen Arbeitgebern, die Forschung und damit auch die Lehre gewinnt durch aktuelle Inhalte und Problemlösungen.

Das diesem Bericht zugrundeliegende Themenfeld „Ausbau der Erneuerbaren Energien " ist beispielhaft für weitere zukunftsrelevante, typischerweise ebenfalls interdisziplinäre Themenfelder wie z.B. Demografie im ländlichen Raum oder Infrastruktur der Zukunft. 
Dementsprechend hat auch die Studienkommission für Hochschuldidaktik an Hochschulen für Angewandte Wissenschaften in Baden-Württemberg ${ }^{19}$ diesen Ansatz als innovatives Lehrprojekt ausgezeichnet und finanziell unterstützt.

\section{Literaturverzeichnis}

Berendt, Brigitte/Voss, Hans-Peter/Wildt, Johannes (Hrsg.), Neues Handbuch Hochschullehre, Bonn 2001.

Bruckert, Felix/Lechleiter, Nele, Windenergie - Steuerungsmöglichkeiten baden-württembergischer Kommunen im Licht der Bürgerakzeptanz, in: VR 2013, S. $374 \mathrm{ff}$.

Franco, Vasili/Damm, Oliver, Netzausbau - Netzinfrastruktur und die Herausforderung der Energiewende, in: VBlBW 2013, S. $327 \mathrm{ff}$.

Franco, Vasili/Frey, Michael, Möglichkeiten zur Zulassung von Windenergieanlagen trotz entgegenstehender Darstellungen in der Flächennutzungsplanung, in: BauR 2014, $1088 \mathrm{ff}$.

Frey, Tina/Staiger, Jonathan, Bioenergiedörfer, in: apf 2014, $299 \mathrm{ff}$.

Fritz, Martin/Moll, Pascal, Die gesicherte Erschließung von Windenergieanlagen auf FNP- und Anlagengenehmigungsebene, in: VR 2014, $338 \mathrm{ff}$.

Herbst, Markus, Entsorgung von Elektroschrott, in: VR 2014, $192 \mathrm{ff.}$

Herbst, Markus, Erneuerbare Energien - Solaranlagen, in: VR 2013, S. $336 \mathrm{ff}$.

Holzapfel, Sandra/Müller, Marco, Erneuerbare Energien - Die Biogasanlage, in: VR 2013, S. $233 \mathrm{ff}$.

Huber, Ludwig, Methodische Anregungen für den Umgang mit pragmatischen Schwierigkeiten im Forschenden Lernen, in: ders./Kröger/Schelhowe (Hrsg.), Forschendes Lernen als Profilmerkmal einer Universität, Beispiele aus der Universität Bremen, Bielefeld 2013, S. 247 ff.

Huber, Ludwig, Warum Forschendes Lernen nötig und möglich ist, in: ders./Hellmer/Schneider (Hrsg.), Forschendes Lernen im Studium. Bielefeld 2009, S. 9 ff.

Kaufmann, Carina/Knobelspies, Fabian, Wasserkraft - Die erneuerbare Energie der Zukunft, in: ZNER 2013, S. $250 \mathrm{ff}$.

Miller, Simon/Reichert, Erik, Kleine Wasserkraft, in: Wassertriebwerk 2014, i.E.

Pusch, Sara/Witzig, Rico, Erneuerbare Energien \& das liebe Geld, in: innovative VERWALTUNG 2013, 9/2013, S. $41 \mathrm{ff}$.

Rothardt, Isabelle/Burkhardt, Isabelle, Dachflächen-Photovoltaikanlagen auf denkmalgeschützten Gebäuden, in: ZfBR 2014, i.E.

Schifferdecker, Julia, Das Spannungsfeld zwischen Windkraft und Artenschutz auf FNP-Ebene, in: NuR 2014, i.E.

Schmidt, Franziska/Roth, Andrea, European Energy Award, in: apf 2014, 299 ff-

Steible, Bernadette/Grothe, Silke, Smart Grids, in: VBlBW 2014, i.E.

Zachmann, Spohie-Theres/Späth, Corinna, Verwertung von Gärresten aus rechtlicher Sicht, in: VR 2014, i.E.

19 http://www.hochschuldidaktik.net/ (29.5.2014). 